\title{
Highly Compact and Scalable Waveguide-Integrated Single Photon Spectrometer Based on Tailored Disorder
}

\author{
W. Hartmann, P. Varytis, K. Busch, and W. H. P. Pernice
}

\begin{abstract}
The understanding of the underlying physics of randomly disordered systems has been subject to many different studies, especially in the field of integrated photonics. It has been shown (Redding B, Liew SF, Sarma R, Cao H: Nat Photonics $7: 746,2013)$ that for certain applications randomly disordered photonic systems have major advantages in comparison to conventional systems. One such application is an on-chip integrated spectrometer. Conventional spectrometer designs are based on ring resonators, so-called arrayed waveguide grating or echelle gratings. Those designs are prone to fabrication errors and exhibit a very large footprint.

Here we utilize the unique properties of randomly oriented scatterers to build a highly broadband and narrow linewidth, yet low footprint on-chip integrated spectrometer. In combination with integrated superconducting nanowire singlephoton detectors (Pernice WHP, Schuck C, Minaeva O, Li M, Goltsman GN, Sergienko AV, Tang HX: Nat Commun 3:1325, 2012) this device will be able to resolve optical spectra on the single photon level which is interesting for instance for single-photon spectroscopy or quantum wavelength multiplexing.
\end{abstract}

\section{References}

1. Redding B, Liew SF, Sarma R, Cao H (2013) Nat Photonics 7:746

2. Pernice WHP, Schuck C, Minaeva O, Li M, Goltsman GN, Sergienko AV, Tang HX (2012) Nat Commun 3:1325

W. Hartmann $(\bowtie) \cdot$ W. H. P. Pernice

Institute of Physics, University of Münster, Münster, Germany

CeNTech - Center for Nanotechnology, University of Münster, Münster, Germany

e-mail: wladick.hartmann@uni-muenster.de

P. Varytis $\cdot$ K. Busch

Institute of Physics, Humboldt-University Berlin, Berlin, Germany

Max-Born Institute, Berlin, Germany

B. Di Bartolo et al. (eds.), Quantum Nano-Photonics, NATO Science for Peace 